\title{
Time Lapse Imaging Techniques for Comparison of Mineralization Dynamics in Primary Murine Osteoblasts and the Late Osteoblast/Early Osteocyte-Like Cell Line MLO-A5
}

\author{
Sarah L. Dallas ${ }^{\text {a }}$ Patricia A. Veno ${ }^{\text {a }}$ Jennifer L. Rosser ${ }^{\mathrm{a}} \quad$ Cielo Barragan-Adjemian ${ }^{\mathrm{a}}$ \\ David W. Rowe ${ }^{b}$ Ivo Kalajzic ${ }^{b} \quad$ Lynda F. Bonewald $^{a}$

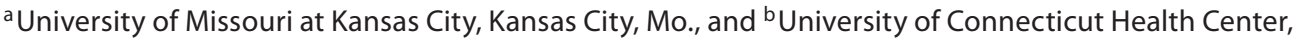 \\ Farmington, Conn., USA
}

\section{Key Words}

Mineralization · Osteoblasts $\cdot$ Osteocytes $\cdot$ Dynamic

imaging $\cdot$ Bone

\begin{abstract}
Mineralization of bone matrix and osteocyte differentiation occur simultaneously and appear interrelated both spatially and temporally. Although these are dynamic events, their study has been limited to using static imaging approaches, either alone or in combination with chemical and biochemical analysis and/or genetic manipulation. Here we describe the application of live cell imaging techniques to study mineralization dynamics in primary osteoblast cultures compared to a late osteoblast/early osteocyte-like cell line, MLOA5. Mineral deposition was monitored using alizarin red as a vital stain for calcium. To monitor differentiation into an osteocyte-like phenotype, the calvarial cells were isolated from transgenic mice expressing green fluorescent protein (GFP) driven by an 8-kb dentin matrix protein-1 (Dmp1) promoter that gives osteocyte-selective expression. Time lapse imaging showed that there was a lag phase of $15-20 \mathrm{~h}$ after $\beta$-glycerophosphate addition, followed by mineral deposition that was rapid in primary osteoblast cultures but more gradual in MLO-A5 cultures. In primary osteoblast cultures,
\end{abstract}

mineral was deposited exclusively in association with clusters of cells expressing Dmp1-GFP, suggesting that they were already differentiating into osteocyte-like cells. In MLO-A5 cells, the first indication of mineralization was the appearance of punctate areas of alizarin red fluorescence of 4-7 $\mu \mathrm{m}$ in diameter, followed by mineral deposition throughout the culture in association with collagen fibrils. A high amount of cell motility was observed within mineralizing nodules and in mineralizing MLO-A5 cultures. These studies provide a novel approach for analyzing mineralization kinetics that will enable us to dissect in a time-specific manner the essential players in the mineralization process.

Copyright $\odot 2008$ S. Karger AG, Basel

\begin{tabular}{ll} 
Abbreviations used in this paper \\
\hline$\alpha$-MEM & minimum essential medium, $\alpha$-modification \\
$\beta$-GP & $\beta$-glycerophosphate \\
DIC & differential interference contrast \\
Dmp1 & dentin matrix protein 1 \\
FBS & fetal bovine serum \\
GFP & green fluorescent protein \\
P/S & penicillin/streptomycin
\end{tabular}

\section{KARGER}

Fax +4161306 1234

E-Mail karger@karger.ch

www.karger.com
(C) 2008 S. Karger AG, Basel

Accessible online at: www.karger.com/cto
Dr. Sarah L. Dallas

School of Dentistry, Department of Oral Biology

University of Missouri at Kansas City, 650 E. 25th Street

Kansas City, MO 64108 (USA)

Tel. +1 816235 6295, Fax +1 816235 5524, E-Mail dallass@umkc.edu 


\section{Introduction}

Mineralization of the osteoid matrix and differentiation of osteoblasts into osteocytes are two processes that occur simultaneously during bone development and formation and appear to be interrelated both spatially and temporally. However, the study of these processes has been largely limited to the use of static imaging approaches, such as light and electron microscopy, either alone or in combination with chemical and biochemical analysis and/or genetic manipulation [Faibish et al., 2005; McKee et al., 2005; Murshed et al., 2005; Huitema and Vaandrager, 2007]. In contrast to static imaging, dynamic molecular imaging approaches enable the visualization of temporal changes in living specimens, such as cells, tissues or embryos, and allow us to quantitate cellular, subcellular and tissue behaviors as a function of time. Dynamic imaging and live cell studies have been successfully used to study morphogenesis during embryonic development and to obtain quantitative insights into various cellular processes as well as into assembly and reorganization of the extracellular matrix [Eils and Athale, 2003; Friedl, 2004; Kulesa, 2004; Sivakumar et al., 2006]. Current technologies, using fluorescent molecular and $\mathrm{pH}$-sensitive dyes or recombinant fluorescent proteins, can label virtually any cellular or extracellular structure. Together with the advancement of imaging techniques such as fluorescence resonance energy transfer, fluorescence recovery after photobleaching and laser confocal microscopy, researchers can obtain biophysical, biochemical, spatial/temporal and kinetic information on cells and subcellular components [for review, see Sekar and Periasamy, 2003; Parsons et al., 2004; Day and Schaufele, 2005; Dallas et al., 2006]. These approaches have significantly enhanced our understanding of many processes fundamental to morphogenesis and development.

In the present study, we have applied live cell imaging approaches to the study of mineralized tissues. In particular, we have examined the dynamic process of mineralization using both primary murine osteoblast cultures and the mineralizing late osteoblast/early osteocyte-like cell line, MLO-A5. Our study highlights the power of these approaches to dissect out, in a spatial and temporal manner, the key steps in the mineralization process and its integration with cell and matrix dynamics in mineralizing tissues.

\section{Materials and Methods}

\section{Cell Culture}

Tissue culture reagents were purchased from Life Technologies Inc. (Gaithersburg, Md., USA) or Mediatech Inc. (Herndon, Va., USA). Primary cultures of neonatal mouse calvarial osteoblasts were isolated from transgenic mice expressing the topaz variant of green fluorescent protein (GFP) under control of an 8-kb fragment of the dentin matrix protein 1 (Dmp1) promoter [Kalajzic et al., 2004]. This transgene has been extensively characterized and shown to give selective expression of GFP in osteocytes, with very low or undetectable expression in osteoblasts [Kalajzic et al., 2004; Yang et al., 2005]. The cells were isolated by sequential trypsin and collagenase digestion as described previously [Dallas et al., 1995] and were plated into coverslip-bottomed Lab-Tek chamber slides at $2 \times 10^{4}$ cells $/ \mathrm{cm}^{2}$ plate area in minimum essential medium, $\alpha$-modification ( $\alpha$-MEM), supplemented with $10 \%$ fetal bovine serum (FBS), $2 \mathrm{mM} \mathrm{L}$-glutamine and $100 \mathrm{U} / \mathrm{ml}$ penicillin/streptomycin $(\mathrm{P} / \mathrm{S})$. At confluence, the medium was changed to $\alpha$-MEM, with additives as above, except that the serum concentration was reduced to $5 \%$ and the media was supplemented with $50 \mu \mathrm{g} / \mathrm{ml}$ ascorbic acid. A low concentration $(0.5 \mathrm{mM})$ of $\beta$-glycerophosphate ( $\beta$-GP) was added for $6-8$ days prior to time lapse imaging. MLO-A5 cells were maintained as described previously [Kato et al., 2001; Barragan-Adjemian et al., 2006]. For mineralization experiments, the cells were plated into coverslip-bottomed Lab-Tek chamber slides at $1.25 \times 10^{4}$ cells $/ \mathrm{cm}^{2}$ in $\alpha$-MEM supplemented with a combination of $5 \%$ FBS, $5 \%$ calf serum and $100 \mathrm{U} / \mathrm{ml} \mathrm{P} / \mathrm{S}$. At confluence, the medium was changed to $\alpha$-MEM supplemented with $10 \% \mathrm{FBS}, 100 \mathrm{U} / \mathrm{ml} \mathrm{P} / \mathrm{S}, 100 \mu \mathrm{g} / \mathrm{ml}$ ascorbic acid and $0.5 \mathrm{mM}$ $\beta$-GP. They were then cultured for 7 days in the presence of 0.5 $\mathrm{mM} \beta$-GP prior to imaging. For both cell culture models, the $\beta$-GP concentration was increased to $4 \mathrm{mM}$ at the start of time lapse imaging and alizarin red $(0.5 \mu \mathrm{g} / \mathrm{ml})$ was added to the culture media as a vital stain for calcium deposition.

\section{Time Lapse Imaging}

Dynamic time lapse imaging was performed on mineralizing primary osteoblast or MLO-A5 cultures using a Nikon TE 2000E deconvolution microscope equipped with wide-field epifluorescence and differential interference contrast (DIC) illumination. The temperature was maintained at $37^{\circ} \mathrm{C}$ using the Cube and Box temperature control system from Life Imaging Services (Reinach, Switzerland), in conjunction with a custom Plexiglas incubation chamber that fully enclosed the microscope and specimen stage. A humidified 5\% $\mathrm{CO}_{2}$ atmosphere was maintained using an automated gas mixer system (The Brick; Life Imaging Services). The microscope system is fully automated, with a precision motorized $\mathrm{x}^{-}, \mathrm{y}-$ and $\mathrm{z}$-stage. The microscope hardware, image acquisition, processing and analysis were controlled by the Metamorph software (Universal Imaging Corporation, West Chester, Pa., USA). $\mathrm{Z}$-stack images were acquired for each time point under $\times 20$ magnification using DIC illumination plus 1 or 2 fluorescent channels. The images were acquired using a Roper Scientific Coolsnap HQ cooled CCD camera with 12-bit grey scale resolution. Fields of $448 \times 335 \mu \mathrm{m}$ were imaged at a spatial resolution of $696 \times 520$ pixels $(2 \times 2$ binned mode $)$ every 15 min for $24-$ $48 \mathrm{~h}$ from 5-7 optical planes. Image stacks were processed in Metamorph using the 'best focus' or Z-projection algorithms and exported as 8-bit image stack files. Red-green merging was per- 
Fig. 1. Representative graphs depicting mineralization kinetics, as measured by alizarin red deposition, in primary cultures of neonatal mouse calvarial cells (a) and MLO-A5 early osteocyte-like cells (b). The alizarin red staining intensity was quantified from time lapse image stacks which were first thresholded to produced binary images, then quantified by particle analysis software (see Materials and Methods).

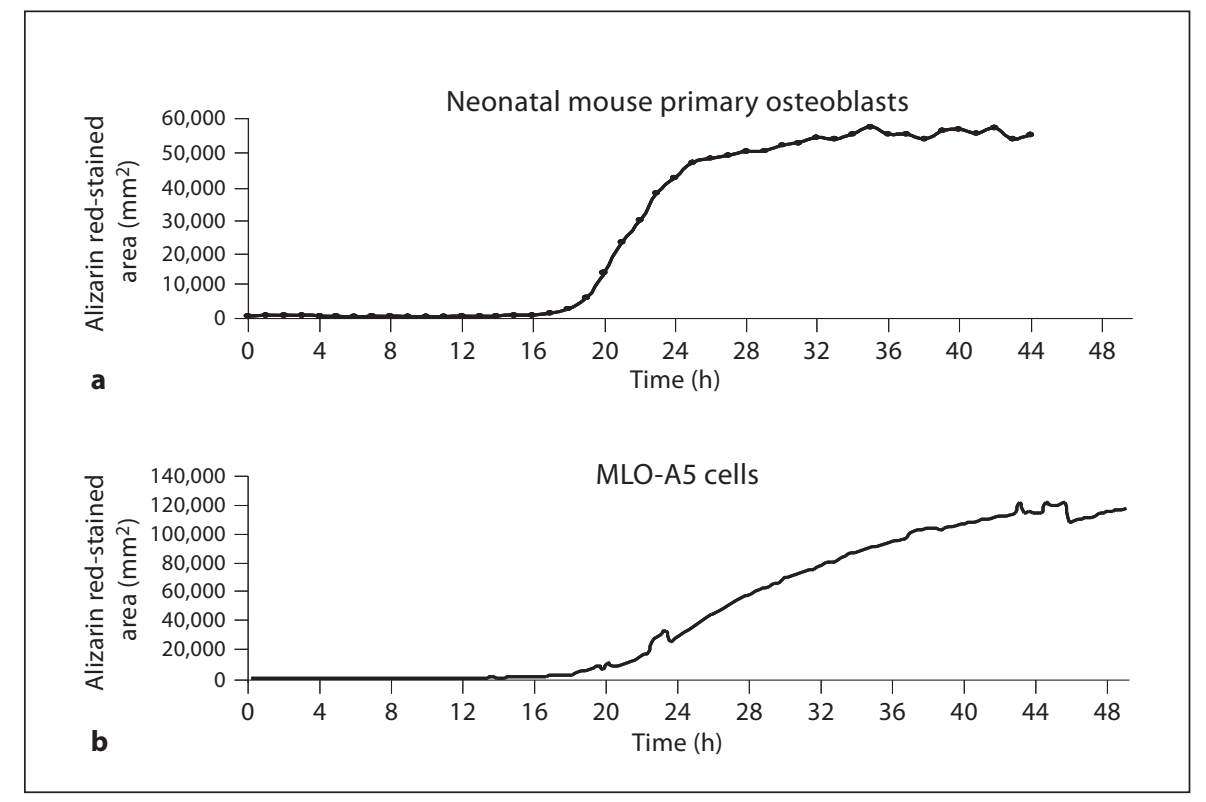

formed using Image J software (http://rsb.info.nih.gov/ij/) and red-DIC merging was performed using the 'red-DIC merge' plugin for Image J (custom written by Bhavik Shah, School of Computing and Engineering, University of Missouri, Kansas City, Mo., USA). Single color or merged image stacks were processed in Image J using the 'stackreg' plugin, which registers/aligns the stack of image slices to correct for rigid body motion [Thevenaz et al., 1998]. Aligned image stacks were assembled into movies using Quicktime Pro or Image J software.

Quantitation of Mineralization Kinetics and Cell Motility

To determine the kinetics of mineralization, aligned image stacks from alizarin red fluorescence images were thresholded in Image J to produce binary images. The area of alizarin red staining in each image frame was then quantified using the 'analyze particles' feature in Image J. To quantitate the motility of Dmp1GFP-positive cells in the primary calvarial cell cultures, individual cell trajectories were plotted frame by frame using the 'MTrackJ' plugin in Image J (Erik Meijering, Biomedical Imaging Group, Erasmus MC - University Medical Center Rotterdam, Rotterdam, The Netherlands). This is a semiautomated analysis in which the operator uses a cursor to plot the position of coordinates representing the center of the cell in successive image frames. The software then calculates the distance and velocity of motion of the cell between successive frames and/or the total distance moved and average velocity.

\section{Results}

Time lapse imaging of alizarin red staining showed that in both primary osteoblast cultures and MLO-A5 cultures, there was a lag phase of 15-20 h after addition of $\beta$-GP that was followed by mineral deposition. The mineral deposition in primary osteoblast cultures was rapid and completed within approximately $15 \mathrm{~h}$ (fig. 1a). In contrast, mineral deposition was more gradual in MLO-A5 cells (fig. 1b). In these cells, the first indication of mineralization was the appearance of small punctate areas of alizarin red fluorescence of about 4-7 $\mu \mathrm{m}$ in diameter (fig. 2b), followed by mineral deposition that occurred throughout the culture in association with collagen fibrils. In contrast, in primary osteoblast cultures, mineral was deposited in discrete nodules and occurred only in association with clusters of cells expressing Dmp1GFP, suggesting that they were already differentiating into osteocyte-like cells (fig. 2a).

In the primary cells, a large amount of membrane ruffling and mitosis preceded mineral deposition. There was also a high degree of cell motility within and around mineralizing nodules as well as in mineralizing MLO-A5 cultures (data not shown). Tracking of the motion trajectories of the Dmp1-GFP-positive cells in the primary osteoblast cultures showed that, overall, the average velocity of the cells was $1.0 \pm 0.2 \mu \mathrm{m} / \mathrm{h}$. However, there appeared to be 2 distinct populations of cells. One population was essentially stationary and there was also a faster moving population of cells that moved at speeds of up to $5 \mu \mathrm{m} / \mathrm{h}$. For these faster moving cells, mineralization was associated with reduced motility, together with the adoption of an osteocyte-like cell morphology in which the cells became less polygonal and more dendritic (data not shown). 

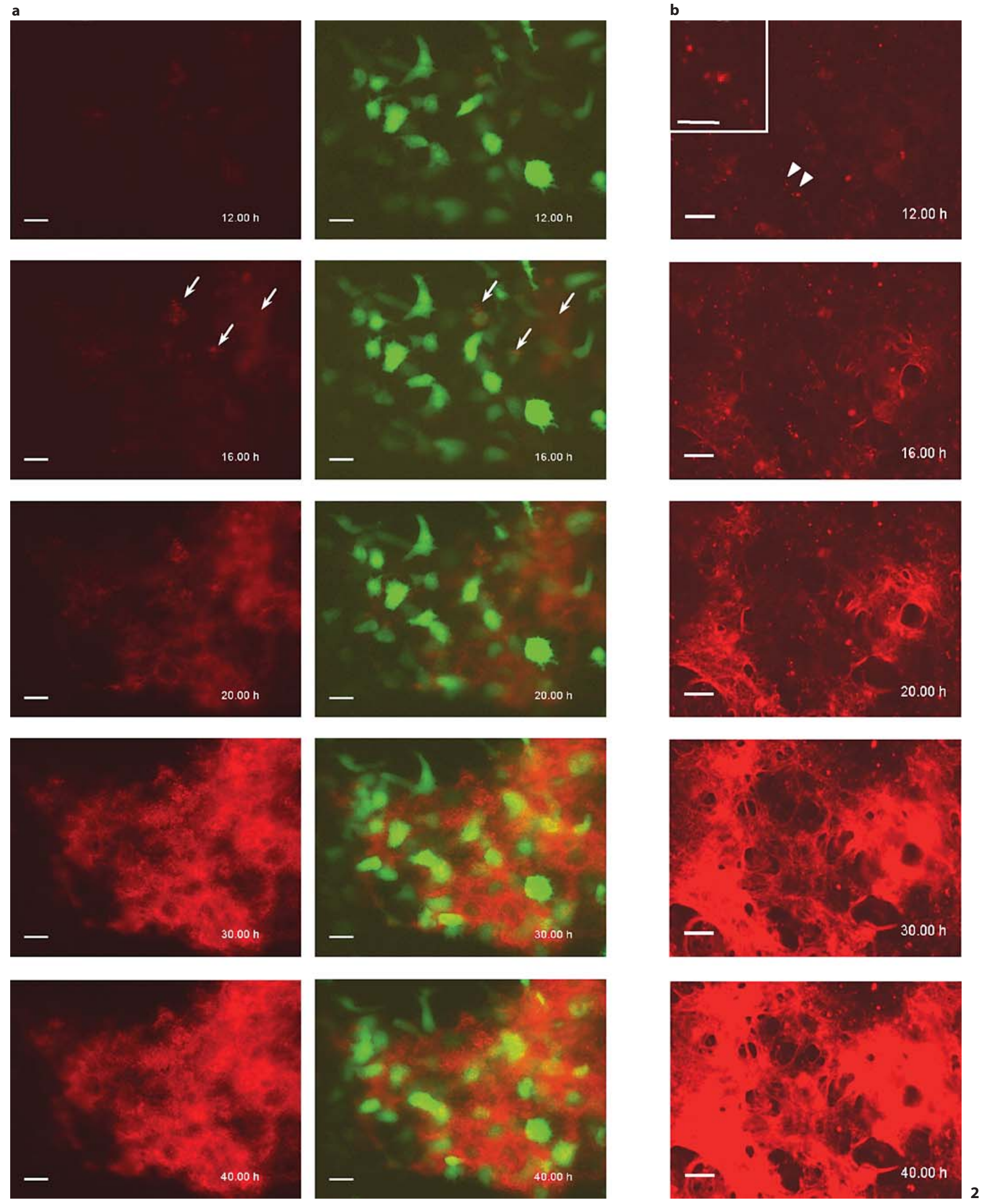


\section{Discussion}

We have presented a novel approach for analyzing mineralization kinetics in vitro, using dynamic time lapse imaging in conjunction with vital staining for calcium deposition as well as lineage markers for differentiation into osteocyte-like cells [Kalajzic et al., 2004]. This novel approach has been used to compare mineralization kinetics in primary mouse osteoblast cultures compared to the late osteoblast/early osteocyte-like cell line, MLO-A5. The same approach would presumably be amenable to the study of mineralization kinetics in cells derived from other mineralizing tissues, such as cartilage and dental tissues, as well as for studying mineralization kinetics in organ culture systems from mineralized tissues.

The processes of mineralization in the primary osteoblast cultures and MLO-A5 cell line, as revealed by time lapse imaging, had many similarities. These include the fact that the mineral in these 2 culture systems appears to be deposited on collagen fibrils and that in both culture systems there was a lag phase of $15-20 \mathrm{~h}$ after addition of $4 \mathrm{mM} \beta$-GP that was followed by mineral deposition. This lag phase is likely due to the time required for processing of the $\beta$-GP by alkaline phosphatase to release inorganic phosphate in a sufficient concentration to initiate mineralization. Future studies comparing mineralization kinetics using inorganic sources of phosphate compared to $\beta$-GP will confirm whether availability of phosphate is responsible for the lag phase in these culture systems. In both culture systems, the mineralizing cell layers also appeared very motile, with a large amount of cell motility, cell division and membrane ruffling activity occurring before and during mineralization (data not shown).

Fig. 2. Representative still image frames from time lapse imaging experiments showing mineralizing cultures of primary mouse osteoblasts from mice expressing GFP driven by the 8-kb Dmp1 promoter (a) and MLO-A5 early osteocyte-like cells (b). a The left column shows alizarin red fluorescence and the right column shows merged images of GFP and alizarin red fluorescence. Note that the initial sites of mineralization (arrows) occur adjacent to GFP-positive cells and that the final pattern of mineral deposition (final frame) corresponds to the location of a large cluster of GFPpositive cells. Scale bars $=25 \mu \mathrm{m}$. b Only alizarin red fluorescence is shown. Note in the 12-hour image that the first indication of mineralization in the MLO-A5 cells is the appearance of small punctuate areas of fluorescence of about 4-7 $\mu \mathrm{m}$ in diameter (arrowheads and inset). This is followed by mineral deposition throughout the culture in association with collagen fibrils. Scale bars $=25 \mu \mathrm{m}$ (inset scale bar $=12.5 \mu \mathrm{m}$ ).
Although there were many similarities between these 2 culture systems, there were also several important differences. For example, the mineral deposition in primary osteoblast cultures was rapid and completed (plateaued) within approximately $15 \mathrm{~h}$. In contrast, mineral deposition appeared to be more gradual and continuous in the MLO-A5 cells. Although the mechanism for this difference in mineralization rate is not yet clear, one possibility is that at the start of the movie, after 7 days in culture, the primary osteoblasts have completed their assembly of extracellular matrix. Therefore, mineral is deposited until all available binding sites are utilized, but then it plateaus. In contrast, the MLO-A5 cells may be able to continue to assemble additional extracellular matrix during the imaging period, which is then mineralized, resulting in a more gradual accumulation of mineral.

Another key difference between these cell models was that mineral deposition in the MLO-A5 cells occurred throughout the culture layer in association with collagen fibrils, a property of a homogeneous cell line representing one stage of osteoblast differentiation. In contrast, in the primary osteoblast cultures, although collagen fibrils are present throughout the osteoblast cell layer (data not shown), mineral deposition was restricted to discrete nodules that invariably contained clusters of cells expressing GFP driven by the 8 -kb Dmp1 promoter. As this transgene is osteocyte selective [Kalajzic et al., 2004; Yang et al., 2005], this suggests that mineralization may be directed by cells that are already differentiating into osteocyte-like cells and/or that specific modifications are made to the collagen within the mineralizing nodule that differentiate it from the collagen in the rest of the cell layer, that is, to prepare or prime it for mineral deposition. Our observations of reduced motility of the Dmp1-GFP-positive cell population, accompanied by a change to a more dendritic cell morphology also suggests that osteocyte differentiation is integrated with mineralization in the primary calvarial osteoblast cultures. In the case of MLOA5, which is a clonal cell line, it could be viewed that all of the cultured cells represent an early osteocyte-like differentiation stage, which could potentially provide an explanation for the fact that mineral deposition occurs throughout the culture.

A further difference between these 2 culture models was that in MLO-A5 cells, the first indication of mineralization was the appearance of small punctate areas of alizarin red fluorescence of about $4-7 \mu \mathrm{m}$ in diameter. These appeared to precede the deposition of mineral onto the collagen fibril network. One possibility is that they are aggregated calcospherulites that act as nucleators for 
mineral deposition on collagen [Barragan-Adjemian et al., 2006]. However, additional studies are needed to further characterize these structures.

Recent studies using time lapse fluorescence microscopy in living cell culture systems have revealed the fact that, far from being a static scaffold, the extracellular matrix is highly dynamic [for review, see Dallas et al., 2006]. A number of different extracellular matrix fibrillar networks have been examined and all have been shown to be highly elastic and undergo a variety of movements and deformations in response to cell motion. In the present study, we have now begun to elucidate some of the cell dynamics that occur during mineralization and have used alizarin red as a vital stain for calcium deposition to reveal the kinetics of mineralization in 2 mineralizing cell models for bone mineralization. Future studies will integrate cell, extracellular matrix and mineralization dynamics to provide novel insights into their spatial and temporal interrelationships in different mineralizing tissues.

In summary, these studies provide a novel approach for analyzing mineralization kinetics that will enable us to dissect in a time-specific manner the essential players in the mineralization process.

\section{Acknowledgements}

This research was supported by NIH grants R21-AR054449 and R01-AR051517 to S.L.D. and PO1 AR046798 to L.F.B. We would also like to acknowledge Drs. Brenda Rongish and Charles Little from the Kansas University Medical Center for expert advice and assistance with dynamic imaging procedures.

\section{References}

Barragan-Adjemian, C., D. Nicolella, V. Dusevich, M.R. Dallas, J.D. Eick, L.F. Bonewald (2006) Mechanism by which MLO-A5 late osteoblasts/early osteocytes mineralize in culture: similarities with mineralization of lamellar bone. Calcif Tissue Int 79: 340 353.

Dallas, S.L., Q. Chen, P. Sivakumar (2006) Dynamics of assembly and reorganization of extracellular matrix proteins. Curr Top Dev Biol 75: 1-24.

Dallas, S.L., K. Miyazono, T.M. Skerry, G.R. Mundy, L.F. Bonewald (1995) Dual role for the latent transforming growth factor-beta binding protein in storage of latent TGF- $\beta$ in the extracellular matrix and as a structural matrix protein. J Cell Biol 131: 539549.

Day, R.N., F. Schaufele (2005) Imaging molecular interactions in living cells. Mol Endocrinol 19: 1675-1686.

-Eils, R., C. Athale (2003) Computational imaging in cell biology. J Cell Biol 161: 477-481.

Faibish, D., A. Gomes, G. Boivin, I. Binderman, A. Boskey (2005) Infrared imaging of calcified tissue in bone biopsies from adults with osteomalacia. Bone 36: 6-12.
Friedl, P. (2004) Dynamic imaging of cellular interactions with extracellular matrix. Histochem Cell Biol 122: 183-190.

Huitema, L.F., A.B. Vaandrager (2007) What triggers cell-mediated mineralization? Front Biosci 12: 2631-2645.

Kalajzic, I., A. Braut, D. Guo, X. Jiang, M.S. Kronenberg, M. Mina, M.A. Harris, S.E. Harris, D.W. Rowe (2004) Dentin matrix protein 1 expression during osteoblastic differentiation, generation of an osteocyte GFP-transgene. Bone 35: 74-82.

Kato, Y., A. Boskey, L. Spevak, M. Dallas, M. Hori, L.F. Bonewald (2001) Establishment of an osteoid preosteocyte-like cell MLO-A5 that spontaneously mineralizes in culture. J Bone Miner Res 16: 1622-1633.

Kulesa, P.M. (2004) Developmental imaging: Insights into the avian embryo. Birth Defects Res C Embryo Today 72: 260-266.

McKee, M.D., W.N. Addison, M.T. Kaartinen (2005) Hierarchies of extracellular matrix and mineral organization in bone of the craniofacial complex and skeleton. Cells Tissues Organs 181: 176-188.

Murshed, M., D. Harmey, J.L. Millan, M.D. McKee, G. Karsenty (2005) Unique coexpression in osteoblasts of broadly expressed genes accounts for the spatial restriction of ECM mineralization to bone. Genes Dev 19: 1093-1104.
Parsons, M., B. Vojnovic, S. Ameer-Beg (2004) Imaging protein-protein interactions in cell motility using fluorescence resonance energy transfer (FRET). Biochem Soc Trans 32: 431-433.

-Sekar, R.B., A. Periasamy (2003) Fluorescence resonance energy transfer (FRET) microscopy imaging of live cell protein localizations. J Cell Biol 160: 629-633.

- Sivakumar, P., A. Czirok, B.J. Rongish, V.P. Divakara, Y.P. Wang, S.L. Dallas (2006) New insights into extracellular matrix assembly and reorganization from dynamic imaging of extracellular matrix proteins in living osteoblasts. J Cell Sci 119: 1350-1360.

Thevenaz, P., U.E. Ruttimann, M. Unser (1998) A pyramid approach to subpixel registration based on intensity. IEEE Trans Image Process 7: 27-41.

Yang, W., Y. Lu, I. Kalajzic, D. Guo, M.A. Harris, J. Gluhak-Heinrich, S. Kotha, L.F. Bonewald, J.Q. Feng, D.W. Rowe, C.H. Turner, A.G. Robling, S.E. Harris (2005) Dentin matrix protein 1 gene cis-regulation: use in osteocytes to characterize local responses to mechanical loading in vitro and in vivo. J Biol Chem 280: 20680-20690. 